\title{
Nondestructive Evaluation for the Viability of Watermelon (Citrullus lanatus) Seeds Using Fourier Transform Near Infrared Spectroscopy
}

\author{
Santosh Lohumi ${ }^{1}$, Changyeun $\mathrm{Mo}^{2}$, Jum-Soon Kang ${ }^{3}$, Soon-Jung Hong ${ }^{4}$, Byoung-Kwan Cho ${ }^{1}$ * \\ ${ }^{1}$ Department of Biosystems Machinery Engineering, College of Agricultural and Life Science, Chungnam National \\ University, 99 Daehak-ro, Yuseong-gu, Daejeon 305-764, Korea \\ ${ }^{2}$ National Academy of Agricultural Science, Rural Development Administration, 88-2 Seodun-dong, Gwonseon-gu, \\ Suwon, Gyeonggi-do 441-100, South Korea \\ ${ }^{3}$ Department of Horticultural Bioscience, Pusan National University, Miryang, 627-706, South Korea \\ ${ }^{4}$ Rural Human Resource Development Center, 212 Hyohaeng-ro, Bongdam-eup, Hwaseong-si, \\ Gyeonggi-do 445-893, South Korea
}

Received: October $5^{\text {th }}, 2013$; Revised: November $4^{\text {th }}, 2013$; Accepted: November $4^{\text {th }}, 2013$

\section{Abstract}

Purpose: Conventional methods used to evaluate seeds viability are destructive, time consuming, and require the use of chemicals, which are not feasible to implement to process plant in seed industry. In this study, the effectiveness of Fourier transform near infrared (FT-NIR) spectroscopy to differentiate between viable and nonviable watermelon seeds was investigated. Methods: FT-NIR reflectance spectra of both viable and non-viable (aging) seeds were collected in the range of $4,000-10,000 \mathrm{~cm}^{-1}(1,000-2,500 \mathrm{~nm})$. To differentiate between viable and non-viable seeds, a multivariate classification model was developed with partial least square discrimination analysis (PLS-DA). Results: The calibration and validation set derived from the PLS-DA model classified viable and non-viable seeds with $100 \%$ accuracy. The beta coefficient of PLS-DA, which represented spectral difference between viable and non-viable seeds, showed that change in the chemical component of the seed membrane (such as lipids and proteins) might be responsible for the germination ability of the seeds. Conclusions: The results demonstrate the possibility of using FT-NIR spectroscopy to separate seeds based on viability, which could be used in the development of an online sorting technique.

Keywords: FT-NIR spectroscopy, Nondestructive discrimination, PLS-DA, Seed aging, Seed viability

\section{Introduction}

Watermelon flesh provides an abundant source of nutrition (vitamins and natural antioxidants), and the seeds are a rich source of oil, similar to soybean and corn. However, watermelon production is prone to being highly affected by the poor germination ability of the seeds.

It is well known that seed viability is one of the most important factors influencing crop production. To improve crop production seed viability must be determined before

\footnotetext{
*Comesponding author: Byoung-Kwan Cho

Tel: +82-42-821-6715; Fax: +82-42-823-6246

E-mail: chobk@cnu.ac.kr
}

planting, as high seed quality is important to produce the healthy seedlings. However, deterioration in the chemical component of the seeds has been observed during the period of storage (also termed "ageing period"). During seed aging, the oxidation of polyunsaturated fatty acid would alter membrane permeability and fluidity and reduce cellular compartimentation, thus leading to change in enzymatic activities (Mazliak, 1983). To date several physiological and biochemical changes that occur during seed deterioration have been used to assess seed quality (McDonald, 1999).

However, conventional methods to assess the germination ability of the seeds usually involve biochemical tests, such as tetrazolium (TZ) or the conductivity test. The tetrazolium 
test based on the relative respiration rate of hydrated dead tissues of the embryo, and detects dehydrogenase enzyme activity as an index of the respiration rate and seed viability (Moore, 1976). However, this method required sample preparation and the use of chemicals, and is destructive, leading to the end use of seed samples. The sinapine or amino acid leakage-based method was developed as a non-destructive single-seed basis seed viability test for Brassica species; however, the seeds require pre-treatment before evaluation (Min, 2000).

Fourier transform near infrared (FT-NIR) spectroscopy is regularly used for both the qualitative and quantitative analysis of agricultural and food products, and presents an alternative to time-consuming, wet-chemical, and destructive techniques. This technique is fast; a sample can be analyzed in a fraction of minute, as it simultaneously collects all light frequencies that are transmitted or reflected back from the sample.

FT-NIR spectroscopy measures the variation in radiation (energy) absorption based on the chemical composition and moisture level of biological materials. Chemical bonds between light atoms (such as $\mathrm{C}-\mathrm{H}, \mathrm{O}-\mathrm{H}$, and $\mathrm{N}-\mathrm{H}$ ) generally have high vibrational frequencies, which result in overtones and combination bands that are detectable in the NIR region, 780-2,500 nm (Osborne et al., 1993). FT-NIR spectroscopy has been widely adopted as a nondestructive and chemical-free technique for the analysis of seeds. Today, NIR is also being applied to detect physical differences in seeds, such as discrimination between viable and empty seeds (Tigabu and Oden, 2002). For instance viable and nonviable Scot's pine (pinus sylvestris) seeds are detected through differences in drying rates (Lsestander and Oden, 2002). The efficiency of NIR spectroscopy has also been shown to have the ability to detect genetically modified soybean seeds (Lee and Choung, 2011).

This study investigated the potential of FT-NIR spectroscopy to differentiate between viable and nonviable (artificially aged) watermelon seeds. We assumed that the chemical changes that occur in cell membrane of the seed during the period of storage (aging) generate different FT-NIR result between the two seed types.

\section{Materials and Methods}

\section{Seed samples}

Watermelon seeds were obtained from the $\mathrm{H}$ seed company in Korea, and were used as samples. A total of 950 (450 viable and 500 non-viable) watermelon seeds were used in this study. The samples were stored at $4^{\circ} \mathrm{C}$ until use.

\section{Artificial aging treatment}

To create the non-viable (aged) seeds, an ageing treatment method (accelerated aging) was applied on a set of 500 seeds. Seeds were watered to allow $20 \%$ moisture content by following the equation;

$$
A W(g)=\frac{\mathrm{ISW}(\mathrm{g}) \times(\% \mathrm{EMS}-\% \mathrm{IMC})}{100-\% \mathrm{EMC}}
$$

where AW = added water, ISW = initial seed weight, EMC= expected moisture content and IMC = initial moisture content.

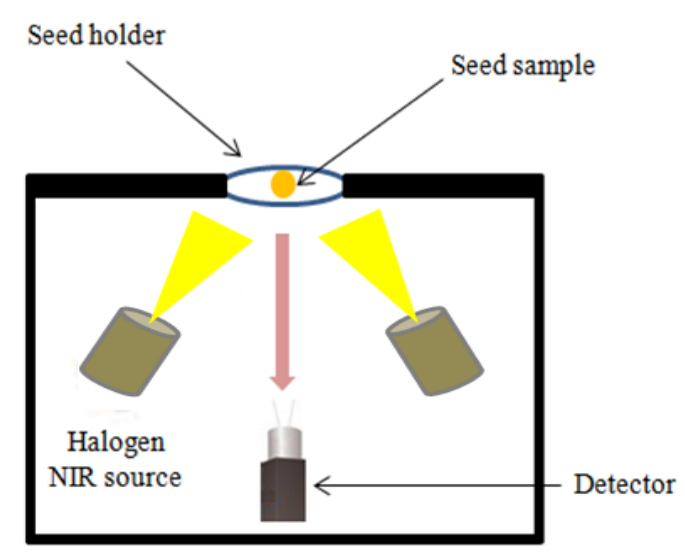

(a)

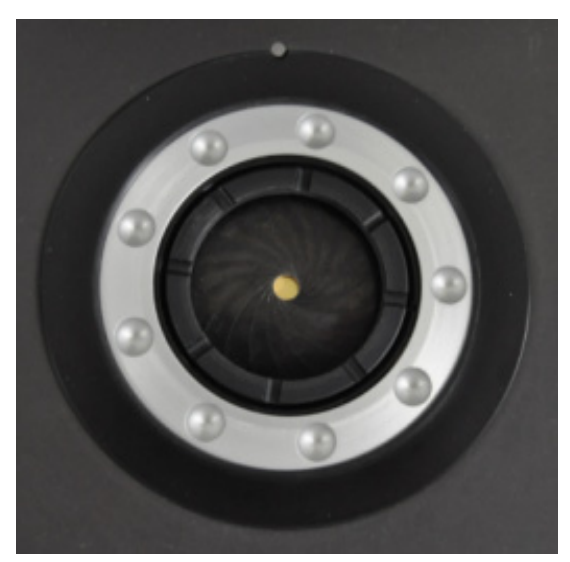

(b)

Figure 1. Schematic of FT-NIR spectroscopy (a) and single-seed holder (b). 
Seeds were vacuumed in a plastic bag and immersed in $50^{\circ} \mathrm{C}$ hot water for 7 days. After 7 days, the seeds were returned to the original weight by drying them at $20^{\circ} \mathrm{C}$ in an incubator.

\section{Acquisition of FT-NIR spectra}

NIR reflectance spectra of 950 seeds were collected on a single-seed basis using an FT-NIR spectrometer (Antaris II FT-NIR analyzer, Thermo Scientific co., USA). The sample holder allows all radiation to reflect back from the surface of the seed (Fig. 1a), as the background of sample holder had a negligible effect with reflected spectra from the seed. Single seeds were placed in a specific sample holder that had a hole in the center, which was of a suitable size and shape for the watermelon seed (Fig. 1b).

The spectra were collected separately from viable and non-viable seeds at a wavelength range between 4,000 and $10,000 \mathrm{~cm}^{-1}(1,000-2,500 \mathrm{~nm})$ at $4 \mathrm{~cm}^{-1}$ interval. The average of 32 successive scans from each seed was obtained, and the mean spectra were used for the analysis. A reference scan was conducted with a golden slit before scanning each sample.

\section{Germination test}

After NIR spectra collection, a germination test was carried out on all seeds to confirm their viability. This validation experiment followed the international seed testing association (ISTA, 1985) standards for seed germination testing. In brief, the seeds were placed between 2 wet anchor seed germination paper and incubated in a germination chamber for 15 days at $25^{\circ} \mathrm{C}$ and $80 \%$ relative humidity. Seeds with a $0.5 \mathrm{~cm}$ visible radical were counted as having germinated, in accordance with ISTA rules.

\section{Data analysis and preprocessing}

The relevant information from the FT-NIR spectroscopy data cannot usually be extracted by direct analysis, due to systemic noise because of light scattering, base line shift, instrumental drift, and slope variation caused by differences in particle size. This type of variation should be removed from the raw spectra so that it does not interfere with useful spectra. To remove unwanted signals, raw spectra were pre-treated using 6 different pre-processing methods, namely, three data normalization methods (minimum, maximum and range) and raw, standard normal variate transformation (SNV), and multiple scatter correlation (MSC). SNV was used to remove slope variation from the spectra caused by scatter and differences in particle size. MSC was used to correct the scatter level of the spectra. The original spectra obtained for the viable and non-viable seeds are depicted in Fig. 2a, while the average spectra of viable and non-viable seeds are presented in Fig. 2 b.

Multivariate classification models were then developed using partial least square discriminant analysis (PLS-DA), using the preprocessed data to classify viable and nonviable seeds. PLS-DA is a potential mathematical approach that

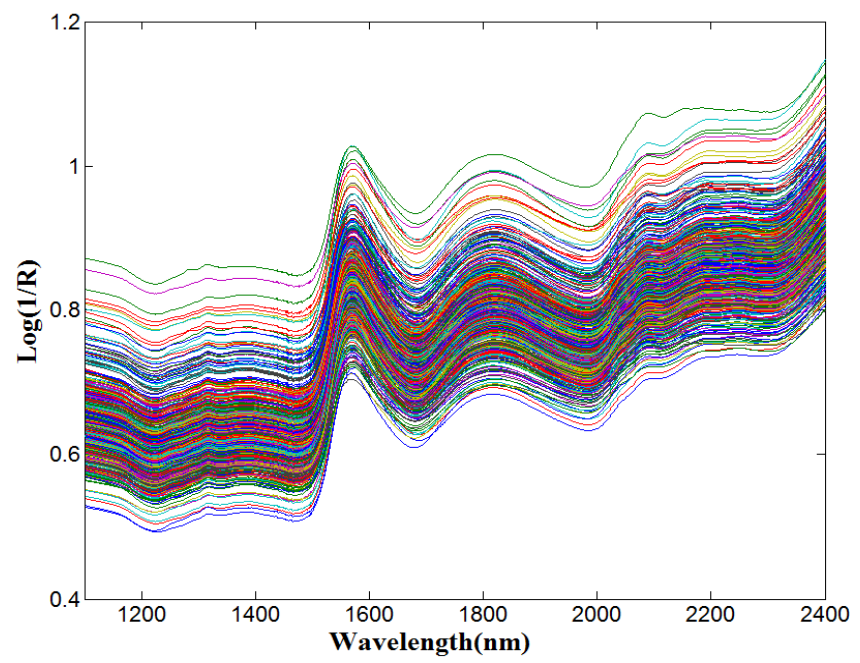

(a)

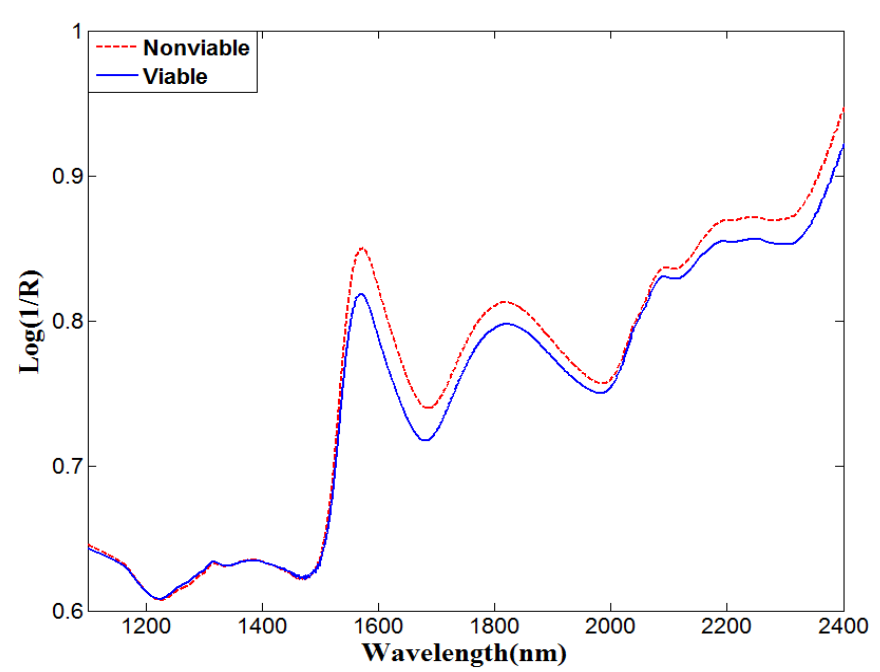

(b)

Figure 2. FT-NIR original spectra of watermelon seeds (a) and mean spectra of the original preprocessed watermelon seeds using the SNV method (b). 
is used for a large number of applications in both qualitative and quantitative analysis. Multivariate methods, such as PLS-DA perform well when we deals with many variables, for instance in spectroscopic applications. Multivariate analysis were perform using MATLAB software version 7.0.4 (The Mathwork, Nitick, MA, USA).

The spectra acquired from the seeds were divided into 2 groups, viable and non-viable (aged), after the germination test. The final classification model was developed using the spectra $(1,100-2,400 \mathrm{~nm})$ from 500 non-viable (aged) seeds and 450 viable seeds. The shorter and longer wavelength region of FT-NIR spectra were removed because they were noisy and contain little useful information. The calibration set was developed with 665 seeds (350 non-viable and 315 viable seeds). The validation efficiency of the fitted PLS-DA model was evaluated using 285 seeds (150 non-viable and 135 viable seeds). The classification rate was defined as the correct value of viable and non-viable seeds predicted by the model in relation to the total number of samples in the test sets.

\section{Results and Discussion}

\section{Evaluation of the PLS-DA model}

The original spectra of viable and non-viable seeds showed little difference in absorption, which might have resulted from differences in the physical and chemical components of the seeds. In addition, there was no specific band that could be used to differentiate the seeds based on viability. The PLS-DA model was developed using preprocessed spectra to classify viable and nonviable seeds. The band pattern for the mean spectra did not show obvious difference between viable and non-viable seeds.

The values of non-viable and viable seeds were artificially set in a y-vector, as 1 and 2 , respectively. The general concept of PLS-DA is that a predicted value of more than 1.5 is counted as viable seeds, a value of less than 1.5 regarded as a non-viable seeds, a value of 1.5 considered as uncertain, which means it may belong to either group. On the basis of the results, the prediction ability of the PLS-DA for both calibration and validation

Table 1. Classification results for the calibration set of the viable and non-viable (aged) watermelon seeds using the PLSDA model with various preprocessing methods

\begin{tabular}{cccccccc} 
& \multicolumn{2}{c}{$\begin{array}{c}\text { Viable seeds } \\
(\mathrm{n}=315)\end{array}$} & \multicolumn{2}{c}{$\begin{array}{c}\text { Non-viable seeds } \\
(\mathrm{n}=350)\end{array}$} & \multicolumn{2}{c}{$\begin{array}{c}\text { Total seeds } \\
(\mathrm{n}=665)\end{array}$} \\
\cline { 2 - 8 } Preprocessing & Correct & Incorrect & Correct & Incorrect & Correct & Incorrect & Accuracy (\%) \\
\hline Min normalization & 315 & 0 & 350 & 0 & 665 & 0 & 100 \\
\hline Max normalization & 315 & 0 & 350 & 0 & 665 & 0 & 100 \\
\hline Range normalization & 315 & 0 & 350 & 0 & 665 & 0 & 100 \\
\hline MSC $^{1)}$ & 315 & 0 & 350 & 0 & 665 & 0 & 100 \\
\hline SNV $^{2)}$ & 315 & 0 & 350 & 0 & 665 & 0 & 100 \\
\hline Raw & 315 & 0 & 350 & 0 & 665 & 0 & 100 \\
\hline
\end{tabular}

Table 2. Prediction results for the validation set of the viable and non-viable (aged) watermelon seeds using the PLSDA model with various preprocessing methods

\begin{tabular}{|c|c|c|c|c|c|c|c|}
\hline \multirow[t]{2}{*}{ Preprocessing } & \multicolumn{2}{|c|}{$\begin{array}{l}\text { Viable seeds } \\
\qquad(n=135)\end{array}$} & \multicolumn{2}{|c|}{$\begin{array}{l}\text { Non-viable seeds } \\
\quad(n=150)\end{array}$} & \multicolumn{3}{|c|}{$\begin{array}{l}\text { Total seeds } \\
(\mathrm{n}=285)\end{array}$} \\
\hline & & Correct & Incorrect & Correct & Incorrect & Correct & Incorrect \\
\hline Min normalization & 135 & 0 & 150 & 0 & 285 & 0 & 100 \\
\hline Max normalization & 135 & 0 & 150 & 0 & 285 & 0 & 100 \\
\hline Range normalization & 135 & 0 & 150 & 0 & 285 & 0 & 100 \\
\hline $\mathrm{MSC}^{\mathrm{a})}$ & 135 & 0 & 150 & 0 & 285 & 0 & 100 \\
\hline$S N V^{b)}$ & 135 & 0 & 150 & 0 & 285 & 0 & 100 \\
\hline Raw & 135 & 0 & 150 & 0 & 285 & 0 & 100 \\
\hline
\end{tabular}

\footnotetext{
a) MSC: Multiple Scatter Correction
}

b) SNV: Standard Normal 
set were $100 \%$ with all preprocessing method. The classification results of the PLS-DA model with each preprocessing method for calibration and validation are shown in Table 1 and 2, respectively.

The result clearly demonstrates that the FT-NIR data contain much information to differentiate between viable and nonviable watermelon seeds. The PLS-DA plot showed the distinct groupings of the viable and nonviable seeds in the calibration and validation sets (Fig. 3).

These results confirm that there are differences between viable and non-viable seeds. Therefore, it is possible to assess seed viability based on chemical component present in reserves of viable seeds. In this study, there was no distinctive difference in the original FT-NIR spectra or appearance between viable and non-viable seeds. This study demonstrates, FT-NIR spectra after pretreatment provide valuable information, with perfect classification being possible using PLS-DA. This method potentially provides an alternative to the currently used destructive, time consuming, and chemical based methods.

\section{Interpretation of the regression coefficient}

The regression coefficient plot (Fig. 4) of PLS-DA indicated that the absorption band contributed to the classification of viable and non-viable watermelon seeds. Differences in energy absorption between these two groups indicate that viable and non-viable seeds had different chemical composition. The main components of watermelon seed are lipids, proteins, and carbohydrates, which accounts for $47 \%, 28 \%$ and $16 \%$ of total seed

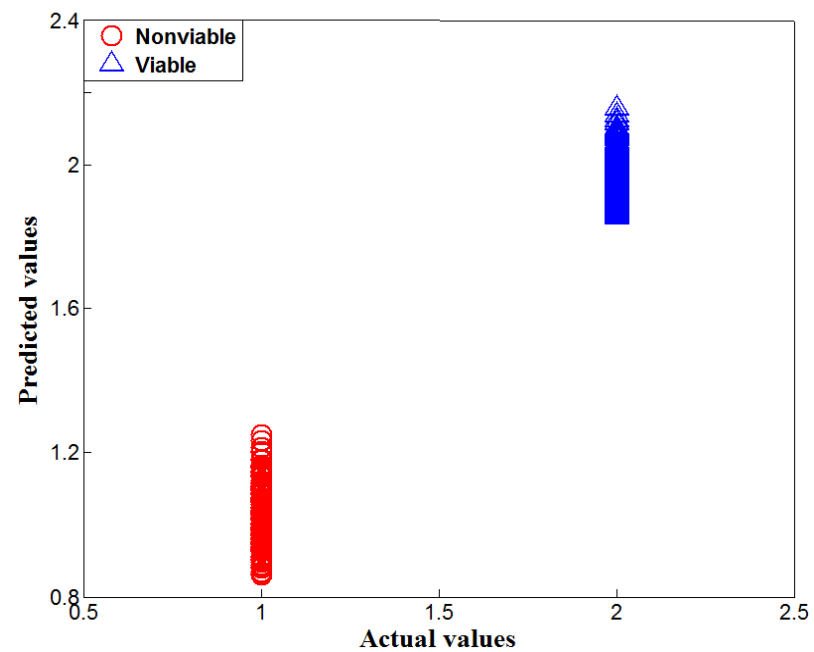

(a) composition respectively. The seed oil contained of 59\% linoleic and 78\% total unsaturated fatty acids (Albishri and Almaghrab, 2013).

The regression coefficient plot indicated the presence of some important absorption peaks. For instance, the 1,150-1,250 region showed a very weak bump around $1,210 \mathrm{~nm}$, which was related to the $\mathrm{C}-\mathrm{H}$ second overtone stretching due to absorption by $\mathrm{CH}_{3}$. The absorption band at $1,570 \mathrm{~nm}$ corresponded to a combination of $\mathrm{N}-\mathrm{H}$ first overtone stretching vibration, which represents protein content. The 1,950-2,050 nm region showed one absorption band near $2,030 \mathrm{~nm}$, which was related to the $\mathrm{C}=0$ second overtone stretching vibration. Several compounds (notably protein, starch, and water) exhibit characteristic absorption in this region (Chung and Kim, 2000). The final part of the spectrum contained an absorption band at 2,252 $\mathrm{nm}$, which corresponded to $\mathrm{O}-\mathrm{H}$ starching $+\mathrm{O}-\mathrm{H}$ deformation, which represent starch. Another absorption band also presents near $2345 \mathrm{~nm}$, which was related to $\mathrm{CH} 2$, this functional group being assigned to lipids.

Thus the reflectance spectra shown by the absorption bands in the current study might represent the characteristics of proteins, carbohydrates, and lipids. Lipid peroxidation caused by free radicals has been suggested to be the main reason for plasma membrane deterioration during seed aging (Wilson and McDonald, 1986). In addition, Lugo and Leopold (1991) observed changes in sugar content during the accelerated aging of maize seeds. Furthermore, protein modification might also contribute to the loss of seed viability.

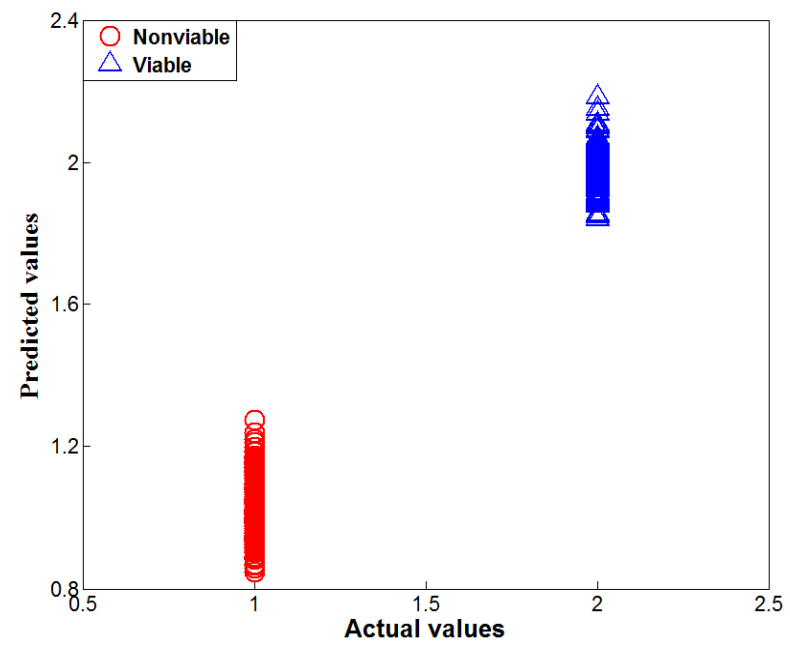

(b)

Figure 3. Classification results of viable and nonviable watermelon seeds using PLS-DA with the maximum normalization of FT-NIR spectra: (a) calibration (b) prediction. 


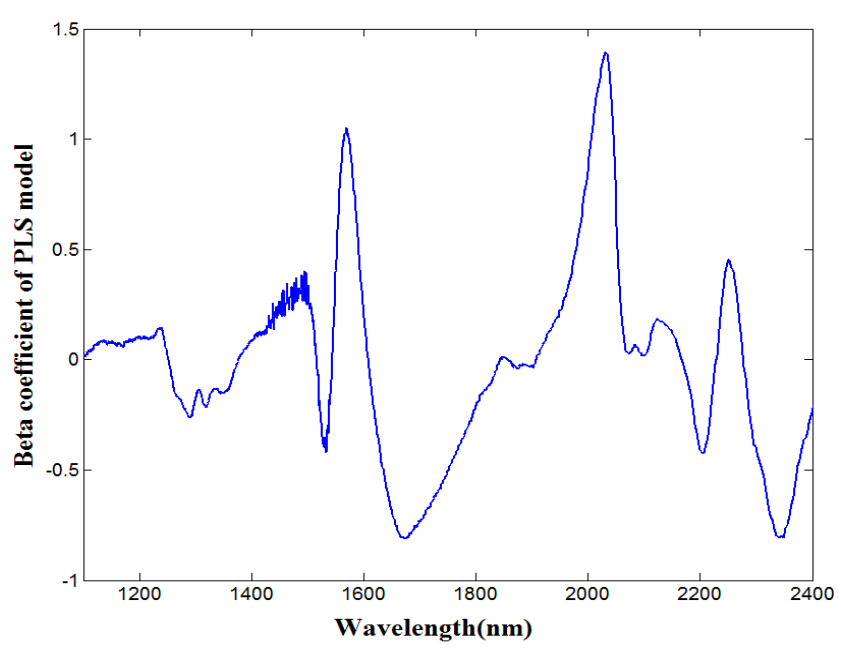

Figure 4. Beta coefficient derived from the PLS-DA model.

\section{Conclusion}

The approach for discriminating viable and aged watermelon seeds using nondestructive spectroscopic technique in combination with a multivariate classification model (PLS-DA) was successful. The calibration and validation set derived from the PLS-DA model classified viable and non-viable seeds with $100 \%$ accuracy. The regression coefficient graph of PLS-DA model proved effectiveness for observing the important wavelength region that best differentiate between viable and nonviable seeds. The FT-NIR measurement technique is rapid, with no required pretreatment, and could potentially automated. Further study is required to classify viable and naturally non-viable seeds using spectroscopic or chemical imaging (hyperspectral imaging) techniques.

\section{Conflict of Interest}

The authors have no conflicting financial or other interests.

\section{Acknowledgement}

This work was supported by Golden Seed Project, Ministry of Agriculture, Food and Rural Affairs (MAFRA), Ministry of Oceans and Fisheries (MOF), Rural Development Administration (RDA) and Korea Forest Service (KFS).

\section{References}

Albishri, H. M. and A. O. Almaghrabi. 2013. Characterization and chemical composition of fatty acid content of watermelon and muskmelon cultivars in Saudi Arabia using gas chromatography/mass spectroscopy 9(33): 58-66.

Chung H. and H. J. Kim. 2000. Near-infrared spectroscopy principles. Analytical science technology 13(1):1-14 (In Korean).

International Seed Testing Association. 1985. International rules for seed testing. Seed science and technology 13:300-520.

Lee, J. H. and M. Choung. 2011. Nondestructive discrimination of herbicide-resistant genetically modified soybean seeds using near-infrared reflectance spectroscopy. Food chemistry 126(1):368-373.

Lugo, I. B. and A. C. Leopold. 1991. Changes in soluble carbohydrates during seed storage. Plant physiology 98(3):1207-1210.

Mazliak, P. 1983. Plant membrane lipids: changes and alterations during aging and senescence. In Liebermann M. (Ed) Postharvest Physiology and Crop Preservation. pp. 123-140, Plenum Press, New York, USA.

McDonald M. B. 1999. Seed deterioration: physiology, repair and assessment. Seed Science and Technology 27(1):177-237.

Min, T. G. 2000. A nondestructive system for detection deteriorated crop seeds by amino acid leakage. J. Kor. Soc. Hort. Sci. 41:576-578.

Moore, R. P. 1976. Tetrazolium seed testing developments in North America. J. Seed Technol. 1:17-30.

Osborne, B. G., T. Fearn and P. T. Hindle. 1993. Practical NIR spectroscopy with applications in food and beverage analysis. 2nd edition. Longman Scientific and Technical, Singapore.

Tigabu, M. and P. C. Oden. 2002. Discrimination of viable and empty seeds of Pinus patula Schiede \& Deppe with NIR spectroscopy. New forest 25:163-176, 2003.

Wilson, D. O. and M. B. McDonald. 1986. The lipid peroxidation model of seed aging. Seed science and technology 14:269-300. 